
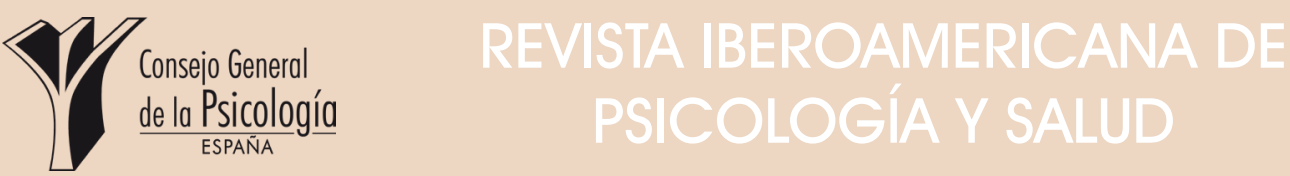

Revista Oficial de la Federación Iberoamericana de Asociaciones de Psicología (FIAP

[Official Journal of the Latin-American Federation of Psychological Associations]

\title{
Revisión sistemática sobre la satisfacción sexual en parejas del mismo sexo
}

\author{
Cristóbal Calvillo', María del Mar Sánchez-Fuentes² y Juan Carlos Sierra ${ }^{\text {* }}$ \\ ${ }^{1}$ Centro de Investigación Mente, Cerebro y Comportamiento (CIMCYC), Universidad de Granada y ${ }^{2}$ Facultad de Ciencias \\ Humanas y Sociales, Universidad de la Costa
}

- Recibido: 02 - 02 - 2018 . Aceptado: 17 - 5 - 2018 . Avance online: 28 - 05 - 2018

RESUMEN: El objetivo principal del presente estudio teórico fue analizar la relación de la satisfacción sexual con variables asociadas a esta en personas adultas con pareja del mismo sexo. Se llevó a cabo una revisión sistemática de investigaciones publicadas que cumplieran con los siguientes criterios de inclusión: (a) evaluasen la satisfacción sexual mediante autoinformes estandarizados o preguntas ad hoc, (b) incluyesen muestras de personas adultas que mantuvieran una relación de pareja del mismo sexo, y (c) relacionasen la satisfacción sexual con variables personales, interpersonales, sociales o ideológico-culturales. Tras una búsqueda bibliográfica a través de diversas bases de datos electrónicas, y una vez realizado un proceso de selección, se presentan los principales resultados de 40 artículos científicos publicados entre 1992 y 2017 . Como resultado más relevante cabe destacar que la satisfacción sexual se asoció con variables de cada una de las dimensiones señaladas. Se revelan más asociaciones con variables personales e interpersonales, y en menor medida con las sociales y culturales. Se discute la importancia de futuras investigaciones en satisfacción sexual en personas con pareja del mismo sexo desde la perspectiva de modelos teóricos consolidados, así como la necesidad de estudios psicométricos en población LGBTI.

PALABRAS CLAVE: Satisfacción sexual, Parejas del mismo sexo, LGBTI, Revisión sistemática.

\section{Systematic review on sexual satisfaction in same-sex couples}

ABSTRACT: The main objective of the present theoretical study was to analyze the relation of sexual satisfaction with variables associated with it in adults with same-sex partners. A systematic review of published research that met the following inclusion criteria was carried out: (a) evaluated sexual satisfaction through standardized self-reports or ad hoc questions, (b) included samples of adults who maintained a samesex relationship, and (c) related sexual satisfaction with personal, interpersonal, social or ideological-cultural variables. After a bibliographic search through various electronic databases, and once a selection process has been carried out, the main results of 40 scientific articles published between 1992 and 2017 are presented. As a most relevant result it should be noted that sexual satisfaction was associated with variables of each of the dimensions indicated. More associations are revealed with personal and interpersonal variables, and to a lesser extent with social and cultural variables. The importance of future research on sexual satisfaction in people with same-sex couple from the perspective of consolidated theoretical models is discussed, as well as the need for psychometric studies in the LGBTI population.

KEYWORDS: Sexual satisfaction, Same-sex couples, LGBTI, Systematic review.

La satisfacción sexual ha sido considerada como un factor determinante de la salud sexual (Henderson, Lehavot y Simoni, 2009), un derecho sexual (Organización Mundial de la Salud, 2010), la última fase del ciclo de la respuesta sexual (Basson, 2001; Sierra y Buela-Casal, 2004) y un componente importante de la calidad de la

*Correspondencia: Juan Carlos Sierra.

Centro de Investigación Mente, Cerebro y Comportamiento

(CIMCYC), Universidad de Granada

Código Postal: 1801 1, Campus Universitario de Cartuja, Granada, España.

E-mail: jcsierra@ugr.es

(C) 2018 Sociedad Universitaria de Investigación en Psicología y Salud. Publicado por Consejo General de Colegios Oficiales de Psicólogos, España. Este es un artículo Open Access relación de pareja (Sprecher, 2002). Lawrance y Byers (1995) la han definido como "una respuesta afectiva derivada de la propia evaluación subjetiva de los aspectos positivos y negativos asociados a las propias relaciones sexuales" (p. 268).

La satisfacción sexual no solo depende de las relaciones sexuales en sí, sino también de otros factores asociados con la relación de pareja, así como de factores socioculturales (Henderson et al., 2009; Sánchez-Fuentes, Salinas y Sierra, 2016; Sánchez-Fuentes, SantosIglesias y Sierra, 2014). La mayoría de los estudios sobre satisfacción sexual se han centrado en 
personas que mantenían una relación de pareja heterosexual (De Jong y Reis, 2015; Mark, García y Fisher, 2015; Sánchez-Fuentes et al., 2016; Sánchez-Fuentes et al., 2014), siendo escasas las investigaciones interesadas que examinan, desde una perspectiva biopsicosociocultural, la satisfacción sexual en parejas del mismo sexo.

Los estudios teóricos son excelentes herramientas que aportan información relevante sobre un problema en cuestión (Checa-Moreno y Quevedo-Blasco, 2017). Por ejemplo, en la revisión sistemática que realizaron SánchezFuentes et al. (2014) sobre satisfacción sexual, la mayoría de los estudios revisados se centraban en personas con pareja de distinto sexo. En dicha revisión se organizaron las variables asociadas a la satisfacción sexual a partir de un modelo integrado por cuatro niveles, propuesto por Henderson et al. (2009): variables personales (e. g., factores biológicos y psicológicos individuales), interpersonales (factores asociados con la relación de pareja), sociales (aspectos relacionados con una comunidad de personas) e ideológicoculturales (e. g., leyes, ideologías y/o tradiciones, etc.). Con el objetivo de ampliar los conocimientos acerca de la satisfacción sexual, el propósito del presente trabajo es realizar la primera revisión sistemática de estudios interesados en variables asociadas a la satisfacción sexual en personas adultas con pareja del mismo sexo. Para ello, se tomará el modelo propuesto por Henderson et al. (2009) para organizar las variables asociadas a la satisfacción sexual.

\section{MÉTODO}

\section{- REVISIÓN DE LA LITERATURA}

La búsqueda de literatura se realizó en las bases de datos Psyclnfo, Scopus y PubMed. Los términos de búsqueda empleados fueron "sexual satisfaction" AND (homosex* OR lesbian* OR gay* OR "same sex"). Todos los términos se buscaron dentro de las categorías Title, Abstract, Keywords, excepto en PubMed que se buscó en All Fields.

\section{• CRITERIOS DE INCLUSIÓN}

Se seleccionaron aquellos artículos que: (a) evaluasen la satisfacción sexual mediante autoinformes estandarizados o preguntas ad hoc, (b) incluyesen muestras de sujetos adultos que mantuvieran una relación de pareja del mismo sexo, y (c) relacionasen la satisfacción sexual con variables personales, interpersonales, sociales o ideológico-culturales. De acuerdo al tipo de documento solo se incluyeron artículos de investigación publicados en inglés o español, sin importar el país de origen donde se llevase a cabo el estudio. No se estableció ninguna limitación en cuanto a la fecha de publicación de los mismos.

\section{- EVALUACIÓN DE LA CALIDAD METODOLÓGICA}

Tras la lectura completa de los artículos que cumplían con los criterios de inclusión se examinó su calidad metodológica empleando la herramienta Quality Assessment Tool for Observational Cohort and Cross-Sectional Studies (National Heart, Lung y Blood Institute, 2014). Este instrumento permite evaluar los artículos con metodología cuantitativa, garantizando su calidad científica y transparencia metodológica mediante la aplicación de una lista de requisitos a cumplir (e. g., "¿̇Estaba la pregunta o el objetivo de investigación claramente establecido en el documento?", "¿Se especificó y definió claramente la población del estudio?").

\section{-PROCEDIMIENTO}

La búsqueda bibliográfica se llevó a cabo entre los meses de noviembre de 2016 y febrero de 2018. De cada artículo que cumplía con los criterios de inclusión se recabó la siguiente información: (a) características de la muestra (número de participantes, sexo y tipo de muestra); (b) instrumento empleado para evaluar la satisfacción sexual; y (c) resultados más importantes obtenidos.

\section{RESULTADOS}

La búsqueda dio lugar a un total de 407 artículos. Tras su análisis para determinar si cumplían los criterios de inclusión y la evaluación de su calidad, quedaron finalmente 40 artículos que fueron objeto de análisis. Véase la Figura 1. La mayoría de artículos excluidos lo fueron porque, aunque incluían muestras de homosexuales, estos no mantenían una relación de pareja con una persona del mismo sexo. Algunos artículos 
BASE DE DATOS: 407 ARTÍCULOS

Excluidos por estar duplicados: 34 artículos

REGISTROS SIN DUPLICADOS

373 ARTICULOS

Excluidos por no ajustarse a los criterios de inclusión: 332 artículos

\section{REGISTROS CON LOS CRITERIOS DE INCLUSIÓN: 41 ARTÍCULOS}

Excluidos por no contar con calidad adecuada: 1 artículo

\section{REGISTROS EVAIUADOS CON} ADECUADA CALIDAD: 40 ARTÍCULOS

no evaluaban la satisfacción sexual en sí, sino que se enfocaban en evaluar aspectos como el funcionamiento sexual, aspectos relacionales con la pareja, actitudes sexuales o dimensiones de la salud en relación con el $\mathrm{VIH}$.

\section{-TIPOS DE MUESTRAS}

Los 40 artículos incluidos en la revisión fueron publicados desde el año 1992 a 2017. Con respecto al tipo de muestra, 25 artículos $(62,50 \%)$ incluían muestras de la población general, tres $(7,50 \%)$ emplearon muestras de población general y estudiantil, nueve $(22,50 \%)$ usaron muestras clínicas (ocho con personas seropositivas y uno incluyó pacientes con cáncer de próstata), y tres artículos $(7,50 \%)$ incluían, como parte de la muestra, población clínica y población general. En cuanto al sexo, 16 artículos (40\%) incluían exclusivamente hombres y $13(32,50 \%)$ mujeres, no encontrándose diferencias entre dichas frecuencias $\left(\chi_{(1)}^{2}=0,31\right.$; $p=0,57)$, y solo 11 artículos $(27,50 \%)$ incluían a ambos sexos. Doce artículos $(30,00 \%)$ contenían muestras de hombres que mantenían una relación de pareja con otro hombre, siete $(17,50 \%)$ a mujeres con una relación de pareja con otra mujer $\left(\chi_{(1)}^{2}=1,31 ; p=0,25\right)$, siete artículos
$(17,50 \%)$ incluían muestras de hombres y mujeres que tenían una relación de pareja con alguien del mismo sexo comparándolos con hombres y mujeres que tenían una relación de pareja con alguien de distinto sexo. De los artículos que incluía muestra homosexual comparándola con muestra heterosexual, seis artículos (15\%) incluían mujeres que tenían una relación homosexual comparándolas con mujeres que tenían una relación heterosexual y cuatro artículos (10\%) incluían hombres que tenían una relación del mismo sexo comparándolos con hombres con una relación heterosexual, no obteniéndose tampoco diferencias entre ambas frecuencias $\left(\chi^{2}{ }_{(1)}=0,40 ; p=0,52\right)$. Por último, tres trabajos $(7,50 \%)$ incluyeron muestras de hombres y mujeres que mantenían una relación de pareja del mismo sexo, y solo un artículo $(2,50 \%)$ incluía muestras de hombres y mujeres que mantenían una relación heterosexual junto con hombres que tenían una relación del mismo sexo.

\section{-INSTRUMENTOS EMPLEADOS}

En cuanto a los instrumentos empleados para evaluar la satisfacción sexual, 30 artículos (75\%) usaron autoinformes estandarizados y diez (25\%) incluyeron preguntas ad hoc, encontrándose diferencias significativas $\left(\chi_{(1)}^{2}\right.$ $=10 ; p<0,01)$. El instrumento de evaluación más empleados fue la Global Measure of Sexual Satisfaction (GMSEX; Lawrance, Byers y Cohen $2011)$, usada en siete artículos $(24,10 \%)$. La subescala de satisfacción sexual de la Brief Sexual Functioning Scale (BSFS; Reynolds III et al., 1988) se utilizó en cuatro artículos (13,80\%). El Index of Sexual Satisfaction (ISS; Hudson, 1998), la versión corta de la New Sexual Satisfaction Scale (NSSS-SF; Štulhofer, Busko y Brouillard, 2011), el Female Sexual Function Index (FSFI; Rosen et al., 2000) y la subescala de satisfacción sexual de la versión larga de la Satisfaction with Life Scale (SWLS; Alfonso, Allison, Rader y Gorman, 1996) fueron medidas usadas en tres artículos, respectivamente (10,30\%). La subescala de satisfacción sexual de la Sexual Functioning Inventory (SFI; Derogatis, 1975) aparece en dos artículos (6,90\%). Finalmente, la subescala de satisfacción sexual de la Multidimensional Sexual Self-Concept Questionnaire (MSSCQ; Snell, 1998), la medida de satisfacción sexual de la Multidimensional Sexuality Questionnaire (MSQ; 
Snell, Fisher y Walters, 1993), la Relationship Interaction Satisfacction Scale (RISS; Buunk, 1990), el Sexual Satisfaction Inventory (SSI; Whitley, 1998), el International Index of Erectile Function (IIEF; Rosen et al., 1997) y el Index of Sex Life (ISL; Chevret, Jaudinot, Sullivan, Marrel y De Gendre, 2004) fueron instrumentos empleados en un solo artículo, respectivamente. Únicamente el estudio de Holmberg y Blair (2009) empleó dos escalas diferentes para evaluar la satisfacción sexual: el Index of Sexual Satisfaction (ISS; Hudson, 1998) y el Sexual Satisfaction Inventory (SSI; Whitley, 1998).

\section{- VARIABLES PERSONALES ASOCIADAS A LA SATISFACCIÓN SEXUAL}

La satisfacción sexual en personas con pareja del mismo sexo fue asociada con la aceptación de la propia homosexualidad de los sujetos. Es decir, niveles bajos de homonegatividad interiorizada (Dupras, 1994; Henderson et al., 2009; Kuyper y Vanwesenbeeck, 2011 ; Rosser, Metz, Bockting y Buroker, 1997; Šević, Ivanković y Štulhofer, 2016 ; Zheng y Zheng, 2017) y una orientación sexual egosintónica (Rosser et al., 1997) se asocian positivamente con la satisfacción sexual.

En cuanto a aspectos relacionados con la salud mental, niveles bajos de ansiedad sexual (Cohen y Byers, 2014; De Ryck et al., 2012), escasa sintomatología psicopatológica (De Ryck et al., 2012; Henderson et al., 2009; SánchezFuentes y Sierra, 2015; Tracy y Junginger, 2007) y buena salud mental (Holmberg, Blair y Phillips, 2010; Remien, Wagner, Dolezal y CarballoDiéguez, 2003; Totenhagen, Butler y Ridley, 2012) se asociaron con elevada satisfacción sexual. En el área de la salud física, no presentar dolor corporal (Sánchez-Fuentes y Sierra, 2015) y disfrutar de buena salud física en general (Holmberg et al., 2010) se vincula a mayor satisfacción sexual, tanto en hombres como en mujeres homosexuales.

Se analizó también el papel de los pensamientos automáticos (Cohen y Byers, 2014). Los pensamientos negativos y no eróticos que experimenta automáticamente la persona durante la actividad sexual se asocian con una baja satisfacción sexual. Presentar compulsión sexual (Starks, Grov y Parsons, 2013), haber sido víctima de abuso sexual infantil (Crump y Byers, 2017; Weingourt, 1998), el abuso de alcohol
(Dolezal, Remien, Wagner, Carballo-Diéguez y Hung, 2015), mayor edad (Biss y Horne, 2005; De Ryck et al., 2012), tener relaciones sexuales sin el consentimiento propio (De Ryck et al., 2012), la preocupación por adquirir una infección de transmisión sexual (De Ryck et al., 2012), tomar fármacos que reducen la libido (De Ryck et al., 2012; Motofei, Rowland, Popa, Kreienkamp y Paunica, 2011 ) y mayor percepción de malestar (Burri et al., 2012), presentar un estilo de apego de tipo ansioso o evitativo (Mark, Patrick y Murray, 2017) son variables asociadas con niveles bajos de satisfacción sexual. Por otra parte, mantener ilusiones sexuales positivas, es decir, tener la percepción de que con la pareja se tienen similitudes y complementariedad (De Jong y Reis, 2015), tener una buena autopercepción del atractivo sexual (Amos y McCabe, 2015) y motivos para tener relaciones sexuales con el fin de mejorar la intimidad con la pareja (Sánchez, Moss-Racusin, Phelan y Crocker, 2011) son variables personales relacionadas con mayor satisfacción sexual.

Por último, actitudes positivas hacia la sexualidad humana se acompañan de mayor satisfacción sexual (Rosser et al., 1997). El rol sexual en hombres y el rol de género en mujeres fueron variables que también se relacionaron con la satisfacción sexual en personas con pareja del mismo sexo. Así, ser activo (insertivo) y versátil se asoció con una alta satisfacción sexual (Zheng y Zheng, 2017). Adoptar un rol femenino o andrógino durante las relaciones sexuales se asoció con alta satisfacción sexual (Rosenzweig y Lebow, 1992).

\section{-VARIABLES INTERPERSONALES ASOCIADAS A LA SATISFACCIÓN SEXUAL}

En cuanto a las variables interpersonales, el buen funcionamiento sexual (Bridges y Horne, 2007; Carvalheira y Costab, 2015; Cohen y Byers, 2014; Henderson et al., 2009), mayor frecuencia de relaciones sexuales (Blair y Pukall, 2014; Bridges y Horne, 2007; Carvalheira y Costab, 2015; Cohen y Byers, 2014; Deenen, Gijs y Van Naerssen, 1994; Farr, Forssell y Patterson, 2010; Rojas Castro, Le Gall, Andreo y Spire, 2010), mayor duración del encuentro sexual (Blair y Pukall, 2014), usar juguetes sexuales (Herbenick, Reece, Sanders, Ghassemi y Fortenberry, 2010) y no practicar comportamientos sexuales de riesgo 
(Gamarel et al., 2014; Starks, Gamarel y Johnson, 2014) se asociaron con alta satisfacción sexual. En mujeres se encontró que las prácticas sexuales no orgásmicas se asociaron con mayor satisfacción sexual (Holmberg y Blair, 2009). También mayor satisfacción sexual se relacionó con la percepción de mayor intimidad emocional y sentimientos de cercanía (Carvalheira y Costab, 2015; Gamarel et al., 2014; Šević et al., 2016; Totenhagen et al., 2012), menor distanciamiento sexual (Deenen et al., 1994), mayor contingencia en la relación (Sánchez et al., 2011), menor incidencia de celos sexuales (Parsons, Starks, Gamarel y Grov, 2012), cohabitar con la pareja (Biss y Horne, 2005; Smith y Horne, 2008; Tracy y Junginer, 2007) y mayor satisfacción con el tipo de acuerdo sexual dentro de la relación de pareja (Carballo-Diéguez, Remien y Wagner, 2000; Hosking, 2014). Además, una alta satisfacción con la relación de pareja (Henderson et al., 2009; Holmberg et al., 2010; Rosser et al., 1997; Sánchez-Fuentes y Sierra, 2015; Tracy y Junginger, 2007) se asoció de manera positiva con la satisfacción sexual. Por el contrario, percibir falta de apoyo por parte de la pareja se relacionó con menor satisfacción sexual (De Ryck et al., 2012). Entre los componentes que integran el Interpersonal Exchange Model of Sexual Satisfaction (IEMSS; Lawrance y Byers, 1995), el balance entre beneficios y costes sexuales, el nivel comparativo entre los beneficios-costes reales y los esperados, y la percepción de igualdad en relación con los beneficios sexuales obtenidos se asociaron con mayor satisfacción sexual (Byers y Cohen, 2017).

\section{-VARIABLES SOCIALES ASOCIADAS A LA SATISFACCIÓN SEXUAL}

Únicamente se encontró que sentirse discriminado por ser seropositivo se asoció con menor satisfacción sexual (De Ryck et al., 2012), mientras que una mayor percepción de apoyo social se relacionó con una alta satisfacción sexual (Henderson et al., 2009).

\section{-VARIABLES IDEOLÓGICO-CULTURALES ASOCIADAS A LA SATISFACCIÓN SEXUAL}

Con respecto a este tipo de variables solamente se halló que la percepción de un mayor bienestar espiritual (Smith y Horne, 2008) se asociaba con mayor satisfacción sexual.
Por último, mediante una prueba de chi cuadrado con las frecuencias de las cuatro variables mencionadas (personales, interpersonales, sociales e ideológico-culturales) se examinó su distribución, evidenciándose diferencias significativas $\left(\chi_{(3)}^{2}=33,05 ; p<\right.$ 0,001 ) en la misma.

\section{DISCUSIÓN}

El objetivo del presente estudio fue realizar una revisión sistemática de las investigaciones que analizaron la satisfacción sexual en parejas adultas del mismo sexo. En los artículos revisados se encontró que, con respecto al sexo de los participantes de los estudios, doce artículos usaron muestras de hombres homosexuales y siete incluyeron muestras de mujeres homosexuales, es decir, hay un menor número de investigaciones con mujeres que con hombres. Aunque esta diferencia no fue significativa, es importante señalar que las investigaciones con parejas de mujeres son más escasas (Cohen y Byers, 2014; Meana, Rakipi, Weeks y Lykins, 2006; Peplau, Fingerhut y Beals, 2004). Ello podría deberse a que la mayoría de investigaciones relacionadas en el área del VIH/SIDA suelen incluir muestras formadas solo por hombres. La población de hombres que tienen sexo con hombres es una de las más afectadas por el VIH en muchas partes del mundo, como es el caso de Estados Unidos (Holloway et al., 2017), México (Fierros, Rivera y Piña, 2011 ), España (Ministerio de Sanidad, Servicios Sociales e lgualdad, 2015), África del Sur y del Este (Ruiseñor-Escudero et al., 2017), Colombia y otras zonas de Latinoamérica (Zea, Olaya, Reisen y Poppen, 2016). Según ONUSIDA (2017), la población de hombres que tienen sexo con hombres (HSH) representó, a nivel global, el $12 \%$ de los nuevos casos de infección por $\mathrm{VIH}$ en el año 2015, en comparación con otras poblaciones de riesgo (trabajadoras del sexo y usuarios de drogas inyectables). Por ello parece lógico pensar que las investigaciones sobre $\mathrm{VIH}$ centren su interés en hombres con parejas del mismo sexo, quedando las mujeres que viven con VIH como una población menos estudiada (De la Rubia, Ramos-Basurtob y Segovia-Chávez, 2015).

Sobre los instrumentos de evaluación utilizados, en la mayoría de estudios revisados se emplearon escalas estandarizadas para evaluar 
la satisfacción sexual, tal como se demostró a nivel estadístico, lo cual consideramos como algo positivo. Sin embargo, únicamente dos de las escalas empleadas están fundamentadas en modelos teóricos de la satisfacción sexual: la Global Measure of Sexual Satisfaction (GMSEX; Lawrance y Byers, 1995) y la New Sexual Satisfaction Scale (NSSS; Štulhofer et al., 2011). Ambos instrumentos son empleados habitualmente tanto en el área de la investigación como en la práctica clínica (Sánchez-Fuentes et al., 2014). No obstante, hay que señalar que ninguna de estas medidas está validada en población homosexual, aunque si muestran adecuada fiabilidad de consistencia interna en muestras de homosexuales. Por tanto, son necesarios futuros estudios psicométricos con el objetivo de adaptar y validar estos instrumentos de evaluación de la satisfacción sexual en población homosexual. En ocasiones, se hacen comparaciones entre poblaciones de distinta orientación sexual sin haber mostrado que las medidas empleadas son invariantes en cuanto a la misma. Tal como señalan Muñiz, Elosúa y Hambleton (2013), se debe disponer de información empírica acerca de la equivalencia del constructo evaluado en diferentes poblaciones. Así pues, examinar la equivalencia de las medidas de satisfacción sexual entre muestras heterosexuales y homosexuales es un reto para futuras investigaciones en esta área.

Por otra parte, esta revisión sistemática evidenció que la satisfacción sexual en parejas del mismo sexo se ha asociado con variables personales, interpersonales, sociales e ideológicoculturales, por lo que esta división propuesta por Henderson et al. (2009), y empleada en su revisión por Sánchez-Fuentes et al. (2014), es aplicable a este tipo de población. Las variables personales e interpersonales han sido las que han suscitado mayor interés al abordar la satisfacción sexual en personas con pareja del mismo sexo, lo que revela su importancia a la hora de explicar la satisfacción sexual en esta población, tal como ocurre en personas heterosexuales (SánchezFuentes et al., 2014). Este conjunto de variables debería tenerse en cuenta en la práctica clínica a la hora de abordar los problemas de satisfacción sexual en personas que mantienen una relación de pareja de tipo homosexual.

En cuanto a las variables personales, los factores relativos a la salud mental se encontraron en un tercio de los artículos revisados (Burri et al., 2012; Cohen y Byers, 2014; De Ryck et al., 2012; Henderson et al., 2009; Holmberg et al., 2010; Remien et al., 2003; SánchezFuentes y Sierra, 2015; Totenhagen et al., 2012; Tracy y Jungingen, 2007). En este sentido, tal como señalan Schönfeld, Brailovskaia, Bieda, Chi Zhang y Margraf (2016), la salud mental se divide en dos dimensiones: positiva (como bienestar general) y negativa (como problemas de salud mental o psicopatologías). Numerosas investigaciones han demostrado que la población homosexual muestra mayor incidencia de problemas de salud mental que la heterosexual (Cochran, Sullivan y Mays, 2003; Gilman et al., 2001; King et al., 2003; McDaniel, Purcell y D'Augelli, 2001 ; Sandfort, de Graaf y Biil, 2003), lo que podría ser consecuencia del estigma social que aún existe en torno a la homosexualidad en muchos ámbitos (Carvajal, 2013; Cochran et al., 2003). Este estigma afectaría, entre otras áreas, a la salud sexual y psicológica. En este sentido, juega un papel importante la homonegatividad interiorizada abordada en seis de los trabajos revisados (Dupras, 1994; Henderson et al., 2009; Kuyper y Vanwesenbeeck, 2011; Rosser et al., 1997; Šević et al., 2016; Zheng y Zheng, 2017). Según el Modelo de Estrés de Minorías de Meyer (2003), el estrés afecta al estado mental de la persona por percibirse estigmatizado a causa de prejuicios, estereotipos y discriminación. Dicho estrés es manifestado, entre otras formas, por la internalización de actitudes sociales negativas. Si esta internalización negativa se asocia con la homofobia es razonable pensar que la persona acabe desarrollando homofobia internalizada, consistente en la autoaceptación de premisas y creencias negativas sobre la homosexualidad (Herek, 2004; Ross y Rosser, 1996). El estrés transformado en homofobia interna ejerce un rol importante en el impedimento de la autoaceptación y el desarrollo de una identidad sexual positiva (Allen y Oleson, 1999; Rowen y Malcolm, 2003), dañando el bienestar personal (Frost, Lehavot y Meyer, 2013; Frost y Meyer, 2009; Meyer y Dean, 1998) y la propia salud sexual. Cabe indicar que la población juvenil homosexual constituye un grupo de riesgo en cuanto a conductas suicidas (Mustanki y Liu, 2013). Teniendo en cuenta que se ha producido un incremento de la conducta suicida en población juvenil (Cano-Montalbán y Quevedo-Blasco, 2018), es conveniente señalar que factores como los síntomas depresivos y 
de ansiedad, la falta de apoyo social por parte de la familia, la desesperanza (Mustanski y Liu, 2013), así como no contar con habilidades de afrontamiento adecuadas o tolerancia a la frustración (Morales et al., 2016) pueden incrementar dicho comportamiento.

Entre las variables interpersonales, la frecuencia sexual (Blair y Pukall, 2014; Bridges y Horne, 2007; Carvalheira y Costab, 2015; Cohen y Byers, 2014; Deenen et al., 1994; Farr et al., 2010; Rojas Castro et al., 2010), el funcionamiento sexual (Bridges y Horne, 2007; Carvalheira y Costab, 2015; Cohen y Byers, 2014; Henderson et al., 2009) y la satisfacción con la relación de pareja (Henderson et al., 2009; Holmberg et al., 2010; Rosser et al., 1997; Sánchez-Fuentes y Sierra, 2015; Tracy y Junginger, 2007) tienen importancia en el nivel de satisfacción sexual en personas con parejas del mismo sexo. La frecuencia sexual en dichas parejas es una cuestión que ha generado controversia. Se sabe que en parejas lésbicas la frecuencia de relaciones sexuales suele ser menor en comparación con parejas de hombres o parejas heterosexuales (Blumstein y Schwartz, 1983; Farr et al., 2010; Peplau y Fingerhut, 2007), lo cual podría ser un reflejo de doble estándar sexual (Moyano, Monge y Sierra, 2017; Sierra, Moyano, VallejoMedina y Gómez-Berrocal, 2018). Aunque estas diferencias también podrían deberse al hecho de que las investigaciones en parejas de mujeres se hayan abordado desde una perspectiva coitocéntrica, es decir, evalúan la frecuencia de las relaciones sexuales teniendo en cuenta la penetración. El hecho de que una mayor frecuencia sexual se haya asociado a más satisfacción sexual podría ser debido a que dicha frecuencia eleva el nivel de receptividad, de deseo y de facilidad para alcanzar el orgasmo (Schwartz y Young, 2009). En cuanto al funcionamiento sexual, este se refiere a la respuesta sexual humana. Un desempeño sexual óptimo conduciría a disfrutar de relaciones sexuales satisfactorias.

La satisfacción con la relación de pareja envuelve aspectos emocionales y relacionales, tales como cohesión emocional, control constructivo de conflictos, consenso y expresión afectiva (Spanier, 1976). Esto supondría que, en una relación satisfactoria de pareja, las relaciones sexuales tendrían un significado más afectivo y emocional, lo que conduciría a mayor satisfacción sexual.

Con respecto a las variables sociales e ideológico-culturales, un reducido número de ellas fueron consideradas en las investigaciones revisadas. Entre las variables sociales, sufrir discriminación por ser seropositivo se asoció con una baja satisfacción sexual (De Ryck et al., 2012), mientras que la percepción de un mayor apoyo social lo hace con mayor satisfacción sexual (Henderson et al., 2009). El apoyo social actúa de antagonista frente al malestar experimentado por la autopercepción de pertenecer a una minoría; sentir apoyo social potencia los sentimientos de autoaceptación de la propia orientación sexual (Doyle y Molix, 2014; Fingerhut, Peplau y Gable, 2010; Frost y Meyer, 2012; Herek, Cogan, Gillis y Glunt, 1998; Thoits, 1995), y para las personas que son seropositivas, dicho apoyo ayuda a paliar los síntomas de trauma relacionados con el virus (Rzeszutek, Oniszczenko, Schier, Biernat-Kałuža y Gasik, 2016). Cabe mencionar que la asociación entre apoyo social percibido y la satisfacción sexual está mediada por la satisfacción con la relación de pareja (Armstrong y Reissing, 2013; Henderson et al., 2009), tal como se demostró también en población heterosexual (SánchezFuentes et al., 2016).

Por último, en las variables ideológicoculturales, únicamente se encontró que un mayor bienestar espiritual se asociaba con mayor satisfacción sexual (Smith y Horne, 2008). Esto puede deberse a que el bienestar espiritual influye sobre la salud física y mental (Stern y Wright, 2017), y dicha salud repercute sobre la satisfacción sexual. Además, la espiritualidad, en comparación con la religión, no se asocia a actitudes homonegativas (Stern y Wright, 2017).

Teniendo en cuenta las características particulares de la relación de pareja entre personas del mismo sexo, se destacan dos variables que se han asociado con la satisfacción sexual en esta población: el rol sexual y el rol de género durante las relaciones sexuales. Con respecto al rol sexual en parejas de hombres, se relacionó el rol activo (insertivo) o versátil con la satisfacción sexual. Ello podría deberse a que asumir un rol pasivo (receptivo) se puede asociar a prácticas receptivas anales dolorosas (Zheng y Zheng, 2017). Mientras que un rol versátil, con la flexibilidad de poder cambiar de rol activo a pasivo (o viceversa) durante las 
relaciones sexuales, constituye un recurso para obtener mayor placer sexual (Zheng y Zheng, 2017). En cuanto al rol de género, en parejas de mujeres se encontró que actuar con un rol más femenino o andrógino en las relaciones sexuales se asoció a más satisfacción sexual (Rosenzweig y Lebow, 1992), hecho que podría relacionarse con los modelos socioculturales que refuerzan a la mujer como sensible y cariñosa (Rosenzweig y Lebow, 1992) durante las relaciones sexuales. Por otro lado, también podría asociarse a conductas relacionadas con la feminidad, como la satisfacción emocional del compromiso y la comunicación durante las relaciones sexuales (Rosenzweig y Dailey, 1989), lo cual conduciría a la satisfacción sexual. Normalmente, estas conductas se presentan más en mujeres que en hombres (Rosenzweig y Dailey, 1989). Esto parece indicar que la satisfacción sexual está más relacionada con ejercer un papel más femenino - andrógino durante las relaciones sexuales, independientemente de la orientación sexual, sexo y género (Rosenzweig y Dailey, 1989).

Se puede concluir que la satisfacción sexual dentro del ámbito de las relaciones homosexuales, al igual que ocurre en las heterosexuales (Sánchez-Fuentes et al., 2014), aparece asociada a variables de tipo personal, interpersonal, social e ideológico-cultural, encontrándose diferencias significativas en la distribución de las frecuencias de dichas variables, manifestando un mayor peso los dos primeros tipos. Así pues, tanto en la práctica clínica como en la investigación es importante tener en consideración estas variables a la hora de comprender mejor la satisfacción sexual en personas con parejas del mismo sexo. Se sugiere para futuras investigaciones profundizar en el estudio de la satisfacción sexual en población LGBTI desde la perspectiva de modelos teóricos consolidados, como por ejemplo el Interpersonal Exchange Model of Sexual Satisfaction (IEMSS; Lawrance y Byers, 1995; Sánchez-Fuentes y Santos-Iglesias, 2016). También son necesarios futuros estudios psicométricos, en población LGBTI, con el fin de conocer la fiabilidad y validez de los instrumentos de evaluación habitualmente empleados en personas heterosexuales para evaluar la satisfacción sexual y, a su vez, demostrar que son medidas invariantes en cuanto a la orientación sexual.

\section{- Conflicto de intereses}

Los autores declaran no tener ningún conflicto de intereses. 
Apéndice 1

Estudios que asocian variables personales, interpersonales, sociales e ideológico-culturales con la satisfacción sexual en personas con pareja del mismo sexo.

\begin{tabular}{|c|c|c|c|c|}
\hline Autores & Participantes & Instrumentos & $\begin{array}{l}\text { Variables asociados } \\
\text { a la satisfacción } \\
\text { sexual }\end{array}$ & $\begin{array}{l}\text { Tipo de } \\
\text { variable }\end{array}$ \\
\hline $\begin{array}{l}\text { Amos y McCabe } \\
\text { (2015) }\end{array}$ & $\begin{array}{l}\text { Población general: hombres } \\
\text { heterosexuales }(n=549), \\
\text { hombres homosexuales } \\
(n=979) \text { y hombres } \\
\text { bisexuales }(n=273) \text {. Mujeres } \\
\text { heterosexuales }(n=468) \\
\text { mujeres homosexuales }(n=246) \\
\text { y mujeres bisexuales }(n=378)\end{array}$ & $\begin{array}{l}\text { Multidimensional } \\
\text { Sexual Self-Concept } \\
\text { Questionnaire } \\
\text { (MSSCQ) }\end{array}$ & $\begin{array}{l}\text { Una percepción } \\
\text { positiva del propio } \\
\text { atractivo (sexual) se } \\
\text { asoció con mayor } \\
\text { satisfacción sexual, } \\
\text { independientemente } \\
\text { de la orientación } \\
\text { sexual }\end{array}$ & Personal \\
\hline Biss y Horne (2005) & $\begin{array}{l}\text { Población general: mujeres } \\
\text { homosexuales }(n=216) \text { y } \\
\text { hombres homosexuales } \\
\qquad(n=360)\end{array}$ & $\begin{array}{l}\text { Versión larga de la } \\
\text { Satisfaction With Life } \\
\text { Scale (SWLS) }\end{array}$ & $\begin{array}{l}\text { En mujeres, mayor } \\
\text { edad se asoció a } \\
\text { menor satisfacción } \\
\text { sexual. } \\
\text { Cohabitar con la } \\
\text { pareja se asoció con } \\
\text { mayor satisfacción } \\
\text { sexual en ambas } \\
\text { muestras }\end{array}$ & $\begin{array}{c}\text { Personal } \\
\text { Interpersonal }\end{array}$ \\
\hline $\begin{array}{l}\text { Blair y Pukall } \\
\text { (2014) }\end{array}$ & $\begin{array}{l}\text { Población general: parejas de } \\
\text { hombres }(n=211) \text {, parejas de } \\
\text { mujeres }(n=215) \text { y parejas } \\
\text { heterosexuales ( } n=396 \text { ) }\end{array}$ & $\begin{array}{l}\text { Dos preguntas para } \\
\text { medir la satisfacción } \\
\text { sexual }\end{array}$ & $\begin{array}{l}\text { Mayor frecuencia } \\
\text { y duración de la } \\
\text { actividad sexual se } \\
\text { asociaron con mayor } \\
\text { satisfacción sexual, } \\
\text { independientemente } \\
\text { de la orientación } \\
\text { sexual }\end{array}$ & Interpersonal \\
\hline $\begin{array}{l}\text { Bridges y Horne } \\
(2007)\end{array}$ & $\begin{array}{l}\text { Población general: mujeres } \\
\text { homosexuales }(N=1.072)\end{array}$ & $\begin{array}{l}\text { Versión larga de la } \\
\text { Satisfaction With Life } \\
\text { Scale (SWLS) }\end{array}$ & $\begin{array}{l}\text { Mejor funcionamiento } \\
\text { sexual (menores } \\
\text { discrepancias en } \\
\text { el deseo sexual) y } \\
\text { mayor frecuencia de } \\
\text { la actividad sexual se } \\
\text { asociaron con niveles } \\
\text { altos de satisfacción } \\
\text { sexual }\end{array}$ & Interpersonal \\
\hline Burri et al. (2012) & $\begin{array}{l}\text { Población general: mujeres } \\
\text { heterosexuales }(n=5.184) \text { y } \\
\text { mujeres homosexuales }(n=814)\end{array}$ & $\begin{array}{c}\text { Female Sexual } \\
\text { Function Index (FSFI) }\end{array}$ & $\begin{array}{l}\text { A mayor malestar } \\
\text { percibido menor } \\
\text { satisfacción sexual }\end{array}$ & Personal \\
\hline
\end{tabular}


Apéndice 1 (Continuación)

Estudios que asocian variables personales, interpersonales, sociales e ideológico-culturales con la satisfacción sexual en personas con pareja del mismo sexo.

\begin{tabular}{|c|c|c|c|c|}
\hline Autores & Participantes & Instrumentos & $\begin{array}{l}\text { Variables asociados a la } \\
\text { satisfacción sexual }\end{array}$ & $\begin{array}{l}\text { Tipo de } \\
\text { variable }\end{array}$ \\
\hline $\begin{array}{c}\text { Byers y } \\
\text { Cohen (2017) }\end{array}$ & $\begin{array}{l}\text { Población general: mujeres } \\
\text { homosexuales }(N=898)\end{array}$ & $\begin{array}{c}\text { Global Measure of } \\
\text { Sexual Satisfaction } \\
\text { (GMSEX) }\end{array}$ & $\begin{array}{l}\text { Percepción de más } \\
\text { beneficios que costes } \\
\text { sexuales, percepción de un } \\
\text { mayor nivel comparativo de } \\
\text { beneficios que de costes } \\
\text { sexuales y percepción de } \\
\text { igualdad de beneficios } \\
\text { sexuales se asociaron con } \\
\text { mayor satisfacción sexual }\end{array}$ & Interpersonal \\
\hline $\begin{array}{l}\text { Carballo- } \\
\text { Diéguez, } \\
\text { Remien y } \\
\text { Wagner, } \\
(2000)\end{array}$ & $\begin{array}{l}\text { Población VIH+: parejas } \\
\text { serodiscordantes de hombres } \\
\qquad(N=63)\end{array}$ & $\begin{array}{l}\text { Brief Sexual } \\
\text { Functioning Scale } \\
\text { (BSFS) }\end{array}$ & $\begin{array}{l}\text { Mayor satisfacción con } \\
\text { el tipo de acuerdo sexual } \\
\text { en la relación de pareja } \\
\text { (monogamia y pareja } \\
\text { abierta), se asoció con } \\
\text { mayor satisfacción sexual }\end{array}$ & Interpersonal \\
\hline $\begin{array}{l}\text { Carvalheira } \\
\text { y Costab } \\
(2015)\end{array}$ & $\begin{array}{l}\text { Población general: hombres } \\
\text { heterosexuales }(n=2.968) \text { y } \\
\text { hombres homosexuales } \\
\qquad(n=285)\end{array}$ & $\begin{array}{l}\text { Versión corta de } \\
\text { la New Scale of } \\
\text { Sexual Satisfaction } \\
\text { (NSSS-SF) }\end{array}$ & $\begin{array}{l}\text { Mejor funcionamiento sexual } \\
\text { y mayor frecuencia de } \\
\text { actividad sexual se asociaron } \\
\text { con más satisfacción sexual, } \\
\text { en la muestra homosexual. } \\
\text { Mayor intimidad emocional } \\
\text { y sentimientos de cercanía } \\
\text { (intimidad en la relación) se } \\
\text { asociaron con una mayor } \\
\text { satisfacción sexual en ambas } \\
\text { muestras }\end{array}$ & Interpersonal \\
\hline $\begin{array}{c}\text { Cohen y } \\
\text { Byers (2014) }\end{array}$ & $\begin{array}{l}\text { Población general: mujeres } \\
\text { homosexuales }(N=586)\end{array}$ & $\begin{array}{c}\text { Global Measure of } \\
\text { Sexual Satisfaction } \\
\text { (GMSEX) }\end{array}$ & $\begin{array}{l}\text { Menor ansiedad } \\
\text { sexual, niveles bajos de } \\
\text { pensamientos automáticos, } \\
\text { mayor deseo sexual y mayor } \\
\text { frecuencia de las relaciones } \\
\text { sexuales de tipo genital, se } \\
\text { asociaron positivamente con } \\
\text { la satisfacción sexual }\end{array}$ & $\begin{array}{c}\text { Personal } \\
\text { Interpersonal }\end{array}$ \\
\hline $\begin{array}{c}\text { Crump y } \\
\text { Byers (2017) }\end{array}$ & $\begin{array}{l}\text { Población general: Mujeres } \\
\text { con pareja del mismo sexo sin } \\
\text { historial de abuso sexual } \\
\text { ( } n=136) \text {. } \\
\text { Población clínica: mujeres con } \\
\text { pareja del mismo sexo con } \\
\text { historial de victimización sexual } \\
\text { en la adolescencia y adultez } \\
\text { ( } n=65 \text { ), mujeres con pareja } \\
\text { del mismo sexo con historial de } \\
\text { abuso sexual infantil limitada } \\
\text { a caricias ( } n=55 \text { ) y mujeres } \\
\text { con pareja del mismo sexo con } \\
\text { historial de abuso sexual infantil } \\
\text { involucrando penetración o } \\
\text { intento de penetración ( } n=43 \text { ) }\end{array}$ & $\begin{array}{c}\text { Global Measure } \\
\text { of Sexual } \\
\text { Satisfaction } \\
\text { (GMSEX) }\end{array}$ & $\begin{array}{l}\text { Presencia de abuso sexual } \\
\text { infantil involucrando } \\
\text { penetración o intento de } \\
\text { penetración, se asoció } \\
\text { con baja satisfacción } \\
\text { sexual }\end{array}$ & Personal \\
\hline
\end{tabular}


Apéndice 1 (Continuación)

Estudios que asocian variables personales, interpersonales, sociales e ideológico-culturales con la satisfacción sexual en personas con pareja del mismo sexo.

\begin{tabular}{|c|c|c|c|c|}
\hline Autores & Participantes & Instrumentos & $\begin{array}{l}\text { Variables asociados a la } \\
\text { satisfacción sexual }\end{array}$ & $\begin{array}{l}\text { Tipo de } \\
\text { variable }\end{array}$ \\
\hline $\begin{array}{l}\text { De Jong y } \\
\text { Reis (2015) }\end{array}$ & $\begin{array}{l}\text { Población general: parejas de } \\
\text { hombres }(n=85) \text { y parejas de } \\
\text { mujeres }(n=162)\end{array}$ & $\begin{array}{l}\text { Global Measure of } \\
\text { Sexual Satisfaction } \\
\text { (GMSEX) }\end{array}$ & $\begin{array}{l}\text { Más pensamientos de } \\
\text { similitud, complementariedad } \\
\text { e ilusiones positivas con la } \\
\text { pareja se asociaron con una } \\
\text { mayor satisfacción sexual en } \\
\text { ambos tipos de parejas }\end{array}$ & Personal \\
\hline $\begin{array}{l}\text { De Ryck et } \\
\text { al. (2012) }\end{array}$ & $\begin{array}{l}\text { Población VIH+: hombres } \\
\text { homosexuales/bisexuales } \\
\text { (n=805) y hombres } \\
\text { heterosexuales } \\
(n=212)\end{array}$ & $\begin{array}{c}\text { Escala de } \\
\text { satisfacción sexual } \\
\text { ad hoc }\end{array}$ & $\begin{array}{l}\text { Mayor edad, síntomas } \\
\text { depresivos, ansiedad, tener } \\
\text { relaciones sexuales sin el } \\
\text { consentimiento propio, } \\
\text { preocupación por adquirir } \\
\text { una infección de transmisión } \\
\text { sexual, tomar ansiolíticos, } \\
\text { falta de apoyo percibido } \\
\text { por parte de la pareja, sufrir } \\
\text { discriminación por ser VIH+ } \\
\text { se asociaron con niveles } \\
\text { bajos satisfacción sexual }\end{array}$ & $\begin{array}{l}\text { Personal } \\
\text { Interpersonal } \\
\text { Social }\end{array}$ \\
\hline $\begin{array}{l}\text { Deenen, } \\
\text { Gijs y Van } \\
\text { Naerssen } \\
\text { (1994) }\end{array}$ & $\begin{array}{l}\text { Población general: hombres } \\
\text { homosexuales }(N=320)\end{array}$ & $\begin{array}{c}\text { Relationship } \\
\text { Interaction } \\
\text { Satisfaction Scale } \\
\text { (RISS) }\end{array}$ & $\begin{array}{l}\text { Mayor frecuencia de } \\
\text { actividad sexual y niveles } \\
\text { bajos de distanciamiento } \\
\text { sexual se asociaron con altos } \\
\text { niveles de satisfacción sexual }\end{array}$ & Interpersonal \\
\hline $\begin{array}{l}\text { Dolezal, } \\
\text { Remien, } \\
\text { Wagner, } \\
\text { Carballo- } \\
\text { Diéguez y } \\
\text { Hung (2005) }\end{array}$ & $\begin{array}{l}\text { Población VIH+: parejas } \\
\text { serodiscordantes de hombres } \\
\qquad(N=117)\end{array}$ & $\begin{array}{l}\text { Brief Sexual } \\
\text { Functioning Scale } \\
\text { (BSFS) }\end{array}$ & $\begin{array}{l}\text { Menor consumo de alcohol } \\
\text { se asoció con niveles altos } \\
\text { de satisfacción sexual }\end{array}$ & Personal \\
\hline $\begin{array}{l}\text { Dupras } \\
\text { (1994) }\end{array}$ & $\begin{array}{l}\text { Población general: hombres } \\
\qquad \begin{array}{l}(n=173) \\
\text { Población VIH+: hombres } \\
(n=88)\end{array}\end{array}$ & $\begin{array}{l}\text { Medida de } \\
\text { Satisfacción } \\
\text { Sexual de la } \\
\text { Multidimensional } \\
\text { Sexuality } \\
\text { Questionnaire }\end{array}$ & $\begin{array}{l}\text { Presentar homofobia } \\
\text { internalizada se asoció con } \\
\text { menor satisfacción sexual }\end{array}$ & Personal \\
\hline $\begin{array}{l}\text { Farr, Forssell } \\
\text { y Patterson } \\
\text { (2010) }\end{array}$ & $\begin{array}{l}\text { Población general: familias } \\
\text { homoparentales ( } n=29 \text { masculinas } \\
\text { y } 27 \text { femeninas) y familias } \\
\text { heteroparentales ( } n=50 \text { ) }\end{array}$ & $\begin{array}{l}\text { Brief Sexual } \\
\text { Functioning Scale } \\
\text { (BSFS) }\end{array}$ & $\begin{array}{l}\text { Mayor frecuencia de } \\
\text { actividad sexual se asoció } \\
\text { con mayor satisfacción } \\
\text { sexual, independientemente } \\
\text { de la orientación sexual }\end{array}$ & Interpersonal \\
\hline
\end{tabular}


Apéndice 1 (Continuación)

Estudios que asocian variables personales, interpersonales, sociales e ideológico-culturales con la satisfacción sexual en personas con pareja del mismo sexo.

\begin{tabular}{|c|c|c|c|c|}
\hline Autores & Participantes & Instrumentos & $\begin{array}{c}\text { Variables asociados } \\
\text { a la satisfacción } \\
\text { sexual }\end{array}$ & $\begin{array}{l}\text { Tipo de } \\
\text { variable }\end{array}$ \\
\hline $\begin{array}{l}\text { Gamarel et al. } \\
\qquad(2014)\end{array}$ & $\begin{array}{l}\text { Población VIH+: parejas } \\
\text { serodiscordantes de hombres } \\
\qquad(N=116)\end{array}$ & $\begin{array}{l}\text { Cuatro preguntas } \\
\text { para medir la } \\
\text { satisfacción sexual }\end{array}$ & $\begin{array}{l}\text { Más intimidad } \\
\text { emocional y } \\
\text { sentimientos de } \\
\text { cercanía (mayor } \\
\text { sensación positiva } \\
\text { de "nosotros" en } \\
\text { la pareja) y menos } \\
\text { comportamientos } \\
\text { sexuales de riesgo se } \\
\text { asociaron con más } \\
\text { satisfacción sexual }\end{array}$ & Interpersonal \\
\hline $\begin{array}{l}\text { Henderson, } \\
\text { Lehavot y Simoni } \\
\text { (2009) }\end{array}$ & $\begin{array}{l}\text { Población general: mujeres } \\
\text { heterosexuales }(n=117) \text { y } \\
\text { homosexuales/bisexuales } \\
\text { ( } n=99) \text {. Población estudiantil: } \\
\text { mujeres heterosexuales }(n=22) \\
\text { y mujeres homosexuales }(n=15)\end{array}$ & $\begin{array}{l}\text { Global Measure of } \\
\text { Sexual Satisfaction } \\
\text { (GMSEX) }\end{array}$ & $\begin{array}{c}\text { Menor } \\
\text { homonegatividad } \\
\text { interiorizada } \\
\text { (baja homofobia } \\
\text { internalizada) se } \\
\text { asoció con una } \\
\text { mayor satisfacción } \\
\text { sexual en muestra } \\
\text { homosexual/bisexual. } \\
\text { Menos síntomas } \\
\text { depresivos, mejor } \\
\text { funcionamiento } \\
\text { sexual y mayor } \\
\text { satisfacción con la } \\
\text { relación de pareja } \\
\text { se asociaron con } \\
\text { índices elevados de } \\
\text { satisfacción sexual en } \\
\text { ambas muestras }\end{array}$ & $\begin{array}{c}\text { Personal } \\
\text { Interpersonal }\end{array}$ \\
\hline $\begin{array}{l}\text { Herbenick Reece, } \\
\text { Sanders, Ghassemi } \\
\text { y Fortenbarry } \\
\text { (2010) }\end{array}$ & $\begin{array}{l}\text { Población general: mujeres } \\
\text { heterosexuales ( } n=1.929) \\
\text { mujeres bisexuales }(n=70) \text { y } \\
\text { mujeres homosexuales }(n=37)\end{array}$ & $\begin{array}{c}\text { Female Sexual } \\
\text { Function Index (FSFI) }\end{array}$ & $\begin{array}{l}\text { Usar juguetes sexuales } \\
\text { se asoció con mayor } \\
\text { satisfacción sexual }\end{array}$ & Interpersonal \\
\hline $\begin{array}{l}\text { Holmberg y Blair } \\
\text { (2009) }\end{array}$ & $\begin{array}{l}\text { Población general: hombres } \\
\text { heterosexuales }(n=48) \text { y } \\
\text { hombres homosexuales }(n=53) \text {. } \\
\text { Mujeres heterosexuales } \\
(n=205) \text { y mujeres } \\
\text { homosexuales } \\
(n=117)\end{array}$ & $\begin{array}{l}\text { Index of Sexual } \\
\text { Satisfaction (ISS) } \\
\text { Sexual Satisfaction } \\
\text { Inventory (SSI) }\end{array}$ & $\begin{array}{c}\text { En mujeres, realizar } \\
\text { prácticas sexuales no } \\
\text { orgásmicas se asoció } \\
\text { con más satisfacción } \\
\text { sexual }\end{array}$ & Interpersonal \\
\hline
\end{tabular}


Apéndice 1 (Continuación)

Estudios que asocian variables personales, interpersonales, sociales e ideológico-culturales con la satisfacción sexual en personas con pareja del mismo sexo.

\begin{tabular}{|c|c|c|c|c|}
\hline Autores & Participantes & Instrumentos & $\begin{array}{c}\text { Variables asociados } \\
\text { a la satisfacción } \\
\text { sexual }\end{array}$ & $\begin{array}{l}\text { Tipo de } \\
\text { variable }\end{array}$ \\
\hline $\begin{array}{l}\text { Holmberg, Blair y } \\
\text { Phillips (2010) }\end{array}$ & $\begin{array}{l}\text { Población general: mujeres } \\
\text { heterosexuales }(n=208) \text { y } \\
\text { mujeres homosexuales } \\
\text { ( } n=114)\end{array}$ & $\begin{array}{l}\text { Index of Sexual } \\
\text { Satisfaction (ISS) }\end{array}$ & $\begin{array}{l}\text { Mejor salud mental, } \\
\text { mejor salud física y } \\
\text { mayor satisfacción } \\
\text { con la relación } \\
\text { de pareja se } \\
\text { asociaron con alta } \\
\text { satisfacción sexual, } \\
\text { independientemente } \\
\text { de la orientación } \\
\text { sexual }\end{array}$ & $\begin{array}{c}\text { Personal } \\
\text { Interpersonal }\end{array}$ \\
\hline Hosking (2014) & $\begin{array}{l}\text { Población general: hombres } \\
\text { homosexuales }(N=772)\end{array}$ & $\begin{array}{l}\text { Tres preguntas para } \\
\text { medir la satisfacción } \\
\text { sexual }\end{array}$ & $\begin{array}{l}\text { Mayor satisfacción } \\
\text { con el tipo de } \\
\text { acuerdo sexual en la } \\
\text { relación de pareja } \\
\text { se asoció con niveles } \\
\text { altos de satisfacción } \\
\text { sexual }\end{array}$ & Interpersonal \\
\hline $\begin{array}{c}\text { Kuyper y } \\
\text { Vanwesenbeeck } \\
\text { (2011) }\end{array}$ & $\begin{array}{l}\text { Población general: mujeres } \\
\text { homosexuales }(n=111), \\
\text { mujeres bisexuales }(n=127) \text { y } \\
\text { mujeres heterosexuales } \\
(n=1.965) \text {. Hombres } \\
\text { homosexuales }(n=84), \text { hombres } \\
\text { bisexuales }(n=74) \text { y hombres } \\
\text { heterosexuales }(n=1.928)\end{array}$ & $\begin{array}{l}\text { Cuatro preguntas } \\
\text { para medir la } \\
\text { satisfacción sexual }\end{array}$ & $\begin{array}{l}\text { Niveles bajos de } \\
\text { homonegatividad } \\
\text { interiorizada se } \\
\text { asociaron con mayor } \\
\text { satisfacción sexual en } \\
\text { muestra homosexual }\end{array}$ & Personal \\
\hline $\begin{array}{l}\text { Mark, Patrick y } \\
\text { Murray (2017) }\end{array}$ & $\begin{array}{c}\text { Población general: hombres } \\
\text { homosexuales }(n=100), \\
\text { hombres heterosexuales } \\
(n=152) \text { y hombres bisexuales } \\
(n=37) . \text { Mujeres homosexuales } \\
(n=67), \text { mujeres heterosexuales } \\
(n=364) \text { y mujeres bisexuales } \\
(n=138)\end{array}$ & $\begin{array}{l}\text { Global Measure of } \\
\text { Sexual Satisfaction } \\
\text { (GMSEX) }\end{array}$ & $\begin{array}{c}\text { Mayor presencia } \\
\text { del estilo de apego } \\
\text { ansioso y evitativo se } \\
\text { asoció con menor } \\
\text { satisfacción sexual, } \\
\text { independientemente } \\
\text { de la orientación } \\
\text { sexual }\end{array}$ & Personal \\
\hline $\begin{array}{l}\text { Motofei, Rowland, } \\
\text { Popa, Kreienkamp } \\
\text { y Paunica (2011) }\end{array}$ & $\begin{array}{l}\text { Pacientes en tratamiento de } \\
\text { cáncer de próstata: hombres } \\
\text { heterosexuales }(n=17) \text { y } \\
\text { hombres homosexuales }(n=12)\end{array}$ & $\begin{array}{l}\text { International Index of } \\
\text { Erectile Function (IIEF) }\end{array}$ & $\begin{array}{l}\text { Los efectos del } \\
\text { fármaco bloqueador } \\
\text { de andrógenos } \\
\text { (biculatamida) se } \\
\text { asociaron con menor } \\
\text { satisfacción sexual }\end{array}$ & Personal \\
\hline
\end{tabular}


Apéndice 1 (Continuación)

Estudios que asocian variables personales, interpersonales, sociales e ideológico-culturales con la satisfacción sexual en personas con pareja del mismo sexo.

\begin{tabular}{|c|c|c|c|c|}
\hline Autores & Participantes & Instrumentos & $\begin{array}{c}\text { Variables asociados } \\
\text { a la satisfacción } \\
\text { sexual }\end{array}$ & $\begin{array}{l}\text { Tipo de } \\
\text { variable }\end{array}$ \\
\hline $\begin{array}{l}\text { Parsons, Starks, } \\
\text { Gamarel y Grov } \\
\text { (2012) }\end{array}$ & $\begin{array}{l}\text { Población general: parejas de } \\
\text { hombres }(N=161)\end{array}$ & $\begin{array}{l}\text { Sexual Functioning } \\
\text { Inventory (SFI) }\end{array}$ & $\begin{array}{l}\text { Niveles bajos de } \\
\text { celos sexuales se } \\
\text { asociaron con mayor } \\
\text { satisfacción sexual }\end{array}$ & Interpersonales \\
\hline $\begin{array}{l}\text { Remien, Wagner, } \\
\text { Dolezal y } \\
\text { Carballo-Diéguez } \\
\text { (2003) }\end{array}$ & $\begin{array}{l}\text { Población } \mathrm{VIH}+\text { : parejas } \\
\text { serodiscordantes de hombres } \\
\qquad(N=75)\end{array}$ & $\begin{array}{l}\text { Brief Sexual } \\
\text { Functioning Scale } \\
\text { (BSFS) }\end{array}$ & $\begin{array}{l}\text { Menor malestar } \\
\text { percibido se } \\
\text { asoció con mayor } \\
\text { satisfacción sexual }\end{array}$ & Personal \\
\hline $\begin{array}{l}\text { Rojas Castro, Le } \\
\text { Gall, Andreo y } \\
\text { Spire (2010) }\end{array}$ & $\begin{array}{l}\text { Población VIH+: hombres que } \\
\text { tienen sexo con hombres } \\
(n=202), \text { hombres heterosexual } \\
\text { es }(n=128) \text { y mujeres } \\
\text { heterosexuales }(n=191)\end{array}$ & $\begin{array}{l}\text { Una pregunta para } \\
\text { medir la satisfacción } \\
\text { sexual }\end{array}$ & $\begin{array}{l}\text { Menor frecuencia de } \\
\text { actividad sexual se } \\
\text { asoció a niveles bajos } \\
\text { de satisfacción sexual }\end{array}$ & Interpersonal \\
\hline $\begin{array}{l}\text { Rosenzweig y } \\
\text { Lebow (1992) }\end{array}$ & $\begin{array}{l}\text { Población general: mujeres } \\
\text { homosexuales }(N=111)\end{array}$ & $\begin{array}{l}\text { Index of Sexual } \\
\text { Satisfaction (ISS) }\end{array}$ & $\begin{array}{l}\text { Tener un rol de } \\
\text { género femenino } \\
\text { o andrógino en las } \\
\text { relaciones sexuales se } \\
\text { asoció con una alta } \\
\text { satisfacción sexual }\end{array}$ & Personal \\
\hline $\begin{array}{c}\text { Rosser, Metz, } \\
\text { Bockting y Buroker } \\
\text { (1997) }\end{array}$ & $\begin{array}{l}\text { Población VIH+: hombres } \\
\text { homosexuales }(N=197)\end{array}$ & $\begin{array}{l}\text { Una pregunta para } \\
\text { medir la satisfacción } \\
\text { sexual }\end{array}$ & $\begin{array}{c}\text { Menor } \\
\text { homonegatividad } \\
\text { interiorizada, niveles } \\
\text { positivos con la } \\
\text { orientación sexual } \\
\text { egosintónica (mayor } \\
\text { confort con la } \\
\text { propia atracción } \\
\text { homosexual), } \\
\text { actitudes positivas } \\
\text { hacia la sexualidad } \\
\text { y mayor satisfacción } \\
\text { con la relación de } \\
\text { pareja se asociaron } \\
\text { con una alta } \\
\text { satisfacción sexual }\end{array}$ & $\begin{array}{c}\text { Personal } \\
\text { Interpersonal }\end{array}$ \\
\hline
\end{tabular}




\section{Apéndice 1 (Continuación)}

Estudios que asocian variables personales, interpersonales, sociales e ideológico-culturales con la satisfacción sexual en personas con pareja del mismo sexo.

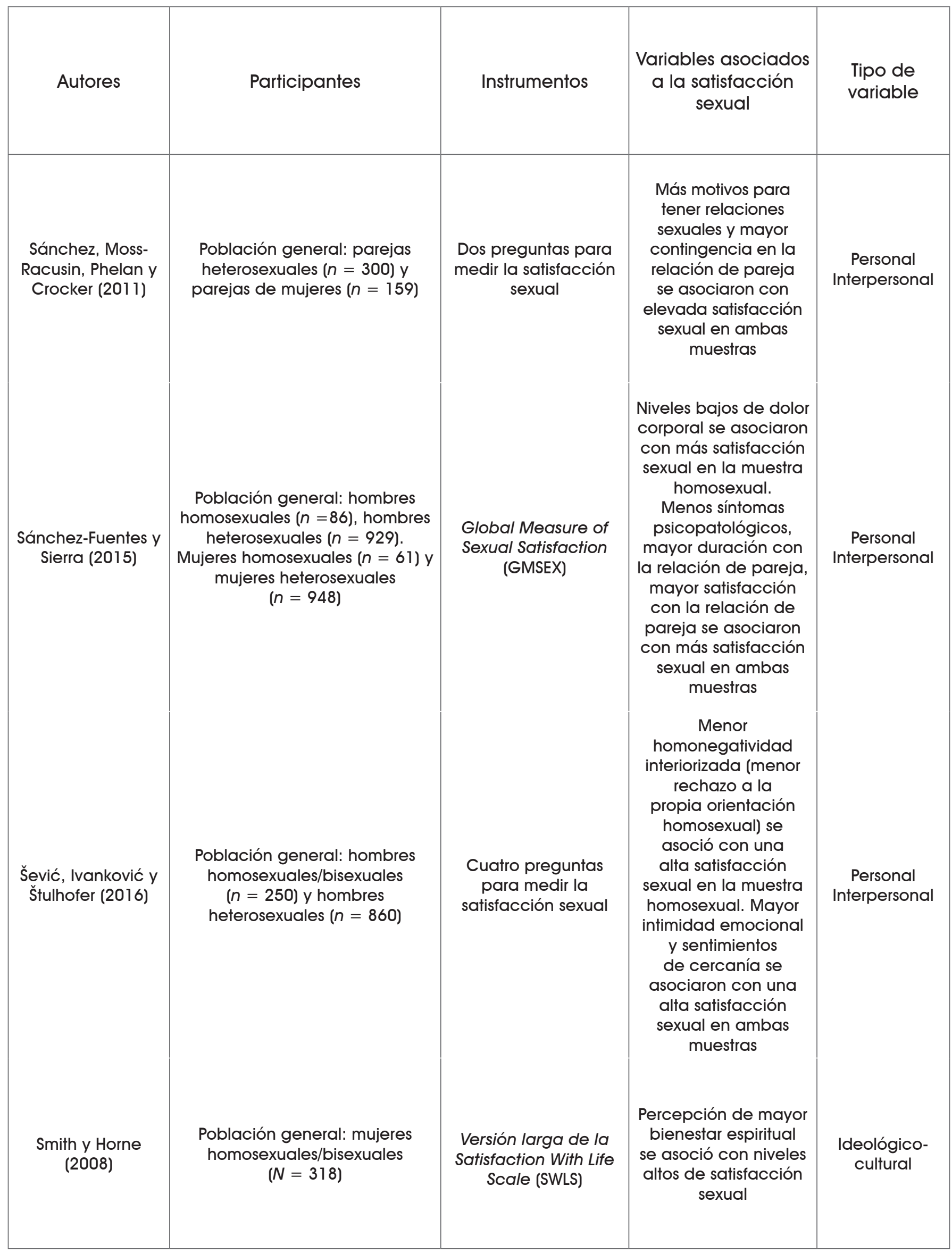




\section{Apéndice 1 (Continuación)}

Estudios que asocian variables personales, interpersonales, sociales e ideológico-culturales con la satisfacción sexual en personas con pareja del mismo sexo.

\begin{tabular}{|c|c|c|c|c|}
\hline Autores & Participantes & Instrumentos & $\begin{array}{c}\text { Variables asociados a la } \\
\text { satisfacción sexual }\end{array}$ & $\begin{array}{l}\text { Tipo de } \\
\text { variable }\end{array}$ \\
\hline $\begin{array}{l}\text { Starks, Gamarel y } \\
\text { Johnson (2014) }\end{array}$ & $\begin{array}{l}\text { Población VIH+: parejas } \\
\text { serodiscordantes de hombres } \\
\qquad(N=91)\end{array}$ & $\begin{array}{l}\text { Cuatro preguntas } \\
\text { para medir la } \\
\text { satisfacción } \\
\text { sexual }\end{array}$ & $\begin{array}{l}\text { Menor frecuencia de } \\
\text { comportamientos sexuales de } \\
\text { riesgo se asoció con niveles } \\
\text { altos de satisfacción sexual }\end{array}$ & $\begin{array}{c}\text { Personal } \\
\text { Interpersonal }\end{array}$ \\
\hline $\begin{array}{l}\text { Starks, Grov y } \\
\text { Parsons (2013) }\end{array}$ & $\begin{array}{l}\text { Población general: parejas } \\
\text { de hombres }(N=172)\end{array}$ & $\begin{array}{c}\text { Sexual } \\
\text { Functioning } \\
\text { Inventory (SFI) }\end{array}$ & $\begin{array}{l}\text { Menor compulsión sexual } \\
\text { se asoció con mayor } \\
\text { satisfacción sexual }\end{array}$ & $\begin{array}{c}\text { Personal } \\
\text { Interpersonal }\end{array}$ \\
\hline $\begin{array}{l}\text { Totenhagen, Butler } \\
\text { y Ridley (2012) }\end{array}$ & $\begin{array}{l}\text { Población general: parejas } \\
\text { de hombres }(n=40) \text { y } \\
\text { parejas de mujeres }(n=55)\end{array}$ & $\begin{array}{l}\text { Una pregunta } \\
\text { para medir la } \\
\text { satisfacción } \\
\text { sexual }\end{array}$ & $\begin{array}{l}\text { Mejor salud mental (niveles } \\
\text { bajos de estrés) y mayor } \\
\text { intimidad emocional y } \\
\text { sentimientos de cercanía se } \\
\text { asociaron con niveles altos } \\
\text { de satisfacción sexual }\end{array}$ & $\begin{array}{c}\text { Personal } \\
\text { Interpersonal }\end{array}$ \\
\hline $\begin{array}{l}\text { Tracy y Junginger. } \\
\text { (2007) }\end{array}$ & $\begin{array}{l}\text { Población general: mujeres } \\
\text { homosexuales }(N=350)\end{array}$ & $\begin{array}{l}\text { Female Sexual } \\
\text { Function Index } \\
\text { (FSFI) }\end{array}$ & $\begin{array}{l}\text { Cohabitar con la pareja } \\
\text { y mayor satisfacción con } \\
\text { la relación de pareja } \\
\text { se asociaron con alta } \\
\text { satisfacción sexual. Más } \\
\text { síntomas psicopatológicos } \\
\text { se asociaron con baja } \\
\text { satisfacción sexual }\end{array}$ & $\begin{array}{l}\text { Ideológico- } \\
\text { cultural }\end{array}$ \\
\hline Weingourt (1998) & $\begin{array}{l}\text { Población sin historial de } \\
\text { abuso sexual infantil: mujeres } \\
\text { homosexuales }(n=12) \text { y } \\
\text { mujeres heterosexuales } \\
(n=36) \text {. Población con } \\
\text { historial de abuso sexual } \\
\text { infantil: mujeres homosexuales } \\
(n=15) \text { y mujeres } \\
\text { heterosexuales }(n=31)\end{array}$ & $\begin{array}{l}\text { Index of Sexual } \\
\text { Satisfaction (ISS) }\end{array}$ & $\begin{array}{l}\text { Ausencia de abuso sexual } \\
\text { infantil se asoció con más } \\
\text { satisfacción sexual }\end{array}$ & Personal \\
\hline $\begin{array}{l}\text { Zheng y Zheng } \\
(2017)\end{array}$ & $\begin{array}{l}\text { Población general: hombres } \\
\text { homosexuales/bisexuales } \\
\text { activos ( } n=56 \text { ), hombres } \\
\text { homosexuales/bisexuales } \\
\text { versátiles ( } n=69 \text { ) y hombres } \\
\text { homosexuales/bisexuales } \\
\text { pasivos ( } n=79 \text { ). Población } \\
\text { estudiantil: hombres } \\
\text { homosexuales/bisexuales } \\
\text { activos ( } n=40 \text { ), hombres } \\
\text { homosexuales/bisexuales } \\
\text { versátiles ( } n=57 \text { ) y hombres } \\
\text { homosexuales/bisexuales } \\
\text { pasivos ( } n=102 \text { ) }\end{array}$ & $\begin{array}{l}\text { Versión corta } \\
\text { de la New } \\
\text { Scale of Sexual } \\
\text { Satisfaction } \\
\text { (NSSS-SF) }\end{array}$ & $\begin{array}{c}\text { Niveles bajos de } \\
\text { homonegatividad } \\
\text { interiorizada y tener un rol } \\
\text { sexual activo se asociaron } \\
\text { con niveles altos de } \\
\text { satisfacción sexual focalizado } \\
\text { a sí mismo }\end{array}$ & Personal \\
\hline
\end{tabular}

NOTA. Fuente: elaboración propia. 


\section{REFERENCIAS}

Alfonso, V.C., Allison. D.B., Rader, D.E. y Gorman, B.S. (1996). The extended satisfaction with life scale: Development and psychometric properties. Social Indicators Research, 38, 275 301. Doi: 10.1007/BF00292049

Allen, D.J. y Oleson, T. (1999). Shame and internalized homophobia in gay men. Journal of Homosexuality, 37, 33-43. doi: 10.1300/ $\mathrm{J} 082 \mathrm{v} 37 \mathrm{n} 0303$

Amos, N. y McCabe, M. (2015). The importance of feeling sexually attractive: Can it predict an individual's experience of their sexuality and sexual relationships across gender and sexual orientation? International Journal of Psychology, 52, 354-363. doi: 10.1002/iiop. 12225

Armstrong, H.L. y Reissing, E.D. (2013). Women who have sex with women: A comprehensive review of the literature and conceptual model of sexual function. Sexual and Relationship Therapy, 28, 364-399. doi:10.1080/146819 94.2013 .807912

Asson, R. (2001). Female sexual response: The role of drugs in the management of sexual dysfunction. Obstetrics \& Gynecology, 98, 350352. Doi: 10.1016/S0029-7844(01)01452-1

Biss, W.J. y Horne, S.G. (2005). Sexual satisfaction as more than a gendered concept: The roles of psychological well-being and sexual orientation. Journal of Constructivist Psychology, 18, 25-38. doi: 10.1080/10720530590523044

Blair, K.L. y Pukall, C.F. (2014). Can less be more? Comparing duration vs. frequency of sexual encounters in same-sex and mixedsex relationships. The Canadian Journal of Human Sexuality, 23, 123-136. doi: 10.3138/ cihs. 2393

Blumstein, P. y Schwartz, P. (1983). American couples: Money, work and sex. Nueva York, NY: William Morrow.

Bridges, S.K. y Horne, S.G. (2007). Sexual satisfaction and desire discrepancy in same sex women's relationships. Journal of Sex \& Marital Therapy, 33, 41-53. doi: $10.1080 / 00926230600998466$

Burri, A., Rahman, Q., Santtila, P., Jern, P., Spector, T. y Sandnabba, K. (2012). The relationship between same-sex sexual experience, sexual distress, and female sexual dysfunction. Journal of Sexual Medicine, 9, 198-206. doi: 10.1111/i.1743-6109.2011.02538.x
Buunk, B. (1990). Relationship interaction satisfaction scale. En J. Touliatos, B.F. Perlmutter y M.A. Strauss (Eds.), Handbook of Family Measurement Techniques (pp. 106-107). Newbury Park, CA: Sage.

Byers, E.S. y Cohen, J.N. (2017). Validation of the interpersonal exchange model of sexual satisfaction with women in a same-sex relationship. PsychologyofWomen Quarterly, 41 , 32-45. doi: $10.1177 / 0361684316679655$

Cano-Montalbán, I. y Quevedo-Blasco, R. (2018). Sociodemographic variables most associated with suicidal behaviour and suicide methods in Europe and America. A systematic review. The European Journal of Psychology Applied to Legal Context, 10, 15-25. doi: 10.5093/ eipalc2018a2

Carballo-Diéguez, A, Remien, R.H. y Wagner, G.J. (2000). Prevalence of extradyadic sex in male couples of mixed HIV status and its relationships to psychological distress and relationship quality. Journal of Homosexuality, 39, 31-46. doi: 10.1300/J082v39n02 02

Carvajal, P. (2013). El reconocimiento de derechos a la comunidad LGBTI. Jurídicas CUC, 9, 123141.

Carvalheira, A.A. y Costab, P.A. (2015). The impact of relational factors on sexual satisfaction among heterosexual and homosexual men. Sexual and Relationship Therapy, 30, 314-324. doi: 10.1080/14681994.2015.1041372

Checa-Moreno, Á. y Quevedo-Blasco, R. (2017). Revisión sistemática en el Síndrome del Acento Extranjero: Intervención y terapia del lenguaje. Revista Iberoamericana de Psicología y Salud, 8, 1-8. doi: 10.23923/i.rips.2017.08.001

Chevret, M., Jaudinot, E., Sullivan, K., Marrel, A. y De Gendre, A.S. (2004). Quality of sexual life \& satisfaction in female partners of men with ED: Psychometric validation of the Index of Sexual Life (ISL) questionnaire. Journal of Sex and Marital Therapy, 30, 141-155. doi: 10.1080/00926230490262339

Cochran, S.D., Sullivan, J.G. y Mays, V.M. (2003). Prevalence of mental disorders, psychological distress, and mental health services use among lesbian, gay, and bisexual adults in the United States. Journal of Consulting and Clinical Psychology, 71, 53-61. doi: 10.1037/0022006X.71.1.53

Cohen, J.N. y Byers, E.S. (2014). Beyond lesbian bed death: Enhancing our understanding of 
the sexuality of sexual-minority women in relationships. Journal of Sex Research, 51, 893903. doi: 10.1080/00224499.2013.795924

Crump, L. y Byers, E.S. (2017). Sexual well-being of sexual minority women in dating relationships who have experienced childhood sexual abuse and/or adolescent and adult victimization. The Canadian Journal of Human Sexuality, 26, 163-173. doi: 10.3138/cihs.262-a4

De Jong, D. y Reis, H. (2015). Sexual similarity, complementarity, accuracy, and overperception in same-sex couples. Personal Relationships, 22, 647-665. doi: 10.1111/pere.12101

De la Rubia, J.M., Ramos-Basurtob, S. y SegoviaChávez, M.P. (2015). Validación cruzada de la escala de expresión de ira en mujeres con VIHy población general. Revista Iberoamericana de Psicología y Salud, 6, 81-89. doi: 10.1016/i. rips.2015.04.003

De Ryck, I. Van Laeken, D., Nöstlinger, C., Platteau, T., Colebunders, R. y The Eurosupport Study Group. (2012). Sexual satisfaction among men living with HIV in Europe. AIDS and Behavior, 16, 225-230. doi: 10.1007/ s10461-011-9987-x

Deenen, A.A., Giis, L. y Van Naerssen, A.X. (1994). Intimacy and sexuality in gay male couples. Archives of Sexual Behavior, 23, 421 431. doi: 10.1007/BF01541407

Derogatis, L.R. (1975). Derogatis Sexual Functioning Inventory (DSFI): Preliminary scoring manual. Baltimore, MD: Clinical Psychometric Research.

Dolezal, C., Remien, R.H., Wagner, G.J., Carballo-Diéguez, A. y Hung, Y. (2005). Alcohol, marijuana, cocaine use, and relationship quality among HIV serodiscordant male couples. The American Journal of Drug and Alcohol Abuse, 31, 593-600. doi: 10.1081/ADA-200068128

Doyle, D.M. y Molix, L. (2014). Perceived discrimination and well-being in gay men: The protective role of behavioural identification. Psychology \& Sexuality, 5, 117-130. doi: 10.1080/19419899.2011.653689

Dupras, A. (1994). Internalized homophobia and psychosexual adjustment among gay men. Psychological Reports, 75, 23-28. doi: 10.2466/pr0.1994.75.1.23

Farr, R.H., Forssell, S.L. y Patterson, C.J. (2010). Gay, lesbian, and heterosexual adoptive parents: Couple and relationship issues.
Journal of GLBT Family Studies, 6, 199-213. doi: 10.1080/15504281003705436

Fierros, L.E., Rivera, B.M. y Piña, J.A. (2011). Sexual behavior with casual partners among university women. Revista Iberoamericana de Psicología y Salud, 2, 165-183.

Fingerhut, A.W., Peplau, L.A. y Gable, S.L. (2010). Identity, minority stress and psychological well-being among gay men and lesbians. Psychology \& Sexuality, 1, 101-114. doi: 10.1080/19419899.2010.484592

Frost, D.M., Lehavot, K. y Meyer, I.H. (2013). Minority stress and physical health among sexual minority individuals. Journal of Behavioral Medicine, 38, 1-8. doi: 10.1007/ s10865-013-9523-8

Frost, D.M. y Meyer, I.H. (2009). Internalized homophobia and relationship quality among lesbians, gay men, and bisexuals. Journal of Counseling Psychology, 56, 97-109. doi: 10.1037/a0012844

Frost, D.M. y Meyer, I.H. (2012). Measuring community connectedness among diverse sexual minority populations. Journal of Sex Research, 49, 36-49. doi: 10.1080/00224499.2011.565427

Gamarel, K.E., Starks, T., Dilworth, S.E., Neilands, T.B., Taylor, J.M. y Johnson, M.O. (2014). Personal or relational? Examining sexual health in the context of HIV serodiscordant same-sex male couples. AIDS and Behavior, 18, 171179. doi: 10.1007/s10461-013-0490-4

Gilman, S.E., Cochran, S.D., Mays, V.M., Hughes, M., Ostrow, D. y Kessler, R.C. (2001). Risk of psychiatric disorders among individuals reporting same-sex sexual partners in the National Comorbidity Survey. American Journal of Public Health, 91, 933-939. Doi: 10.2105/ AJPH.91.6.933

Henderson, A.W., Lehavot, A.K. y Simoni, E.J.M. (2009). Ecological models of sexual satisfaction among lesbian/bisexual and heterosexual women. Archives of Sexual Behavior, 38, 5065. doi: 10.1007/s10508-008-9384-3

Herbenick, D., Reece, M., Sanders, S.A., Ghassemi, A. y Fortenberry, J.D. (2010). Women's vibrator use in sexual partnerships: Results from a nationally representative survey in the United States. Journal of Sex \& Marital Therapy, 36, 49-65. doi: 10.1080/00926230903375677

Herek, G.M. (2004). Beyond "homophobia": Thinking about sexual prejudice and stigma 
in the twenty-first century. Sexuality Research \& Social Policy, 1, 6-24. doi: 10.1525/ srsp.2004.1.2.6

Herek, G.M., Cogan, J.C., Gillis, J.R. y Glunt, E.K. (1998). Correlates of internalized homophobia in a community sample of lesbians and gay men. Journal of the Gay and Lesbian Medical Association, 2, 17-25.

Holmberg, D. y Blair, K.L. (2009). Sexual desire, communication, satisfaction, and preferences of men and women in same-sex versus mixedsex relationships. Journal of Sex Research, 46, 57-66. doi: 10.1080/00224490802645294

Holmberg, D., Blair, K.L. y Phillips, M. (2010). Women's sexual satisfaction as a predictor of well-being in same-sex versus mixed-sex relationships. Journal of Sex Research, 47, 1-11. doi: 10.1080/00224490902898710

Holloway, I.W., Tan, D., Dunlap, S.L., Palmer, L., Beougher, S. y Cederbaum, J.A. (2017). Network support, technology use, depression, and ART adherence among HIV-positive MSM of color. AIDS Care, 29, 1153-1161. doi: $10.1080 / 09540121.2017 .1325435$

Hosking, W. (2014). Australian gay men's satisfaction with sexual agreements: The roles of relationship quality, jealousy, and monogamy attitudes. Archives of Sexual Behavior, 43, 823832. doi: 10.1007/s10508-013-0197-7

Hudson, W.W. (1998). Index of sexual satisfaction. En C.M. Davis, W.L. Yarber, R. Bauserman, G. Schreer y S.L. Davis (Eds), Handbook of sexuality related measures (pp. 512-513). Londres: Sage.

King, M., McKeown, E., Warner, J., Ramsay, A., Johnson, K., Cort, C... Davidson, O. (2003). Mental health and quality of life of gay men and lesbians in England and Wales: Controlled, cross-sectional study. British Journal of Psychiatry, 183, 552-558.

Kuyper, L. y Vanwesenbeeck, I. (2011). Examining sexual health differences between lesbian, gay, bisexual, and heterosexual adults: The role of sociodemographics, sexual behavior characteristics, and minority stress. Journal of Sex Research, 48, 263-274. doi: 10.1080/00224491003654473

Lawrance, K. y Byers, E.S. (1995). Sexual satisfaction in long-term heterosexual relationships: The interpersonal exchange model of sexual satisfaction. Personal Relationships, 2, 267-285. doi: $10.1111 /$ i.1475-6811.1995.tb00092.x
Lawrance, K., Byers, E.S. y Cohen, J.N. (2011). Interpersonal exchange model of sexual satisfaction questionnaire. En T.D. Fisher, C.M. Davis, W.L. Yarber y S.L. Davis (Eds.), Handbook of sexuality related measures ( $3^{a}$ ed., pp. 525 530). Nueva York, NY: Routledge.

Mark, K., García, J. y Fisher, H. (2015). Perceived emotional and sexual satisfaction across sexual relationship contexts: Gender and sexual orientation differences and similarities. The Canadian Journal of Human Sexuality, 24, 120-130. doi: 10.3138/cihs.242-A8.

Mark, K.P., Patrick, L.M. y Murray, S. H. (2017). The impact of attachment style on sexual satisfaction and sexual desire in a sexually diverse sample. Journal of Sex \& Marital Therapy, 22, 1-9. doi: 10.1080/0092623X.2017.1405310

McDaniel, J., Purcell, D. y D'Augelli, A.R. (2001). The relationship between sexual orientation and risk for suicide: Research findings and future directions for research and prevention. Suicide and Life-Threatening Behavior, 31 (Supl), 84-105.

Meana, M., Rakipi, R.S., Weeks, G. y Lykins, A. (2006). Sexual functioning in a non-clinical sample of partnered lesbians. Journal of Couple and Relationship Therapy, 5, 1-22. doi: 10.1300/J398v05n02 01

Meyer, I.H. (2003). Prejudice, social stress, and mental health in lesbian, gay, and bisexual populations: Conceptual issues and research evidence. Psychological Bulletin, 129, 674 697. doi: 10.1037/0033-2909.129.5.674

Meyer, I.H. y Dean, L. (1998). Internalized homophobia, intimacy, and sexual behavior among gay and bisexual men. En G. M. Herek (Ed.), Stigma and sexual orientation: Understanding prejudice against lesbians, gay men, and bisexuals (pp. 160-186). Thousand Oaks, CA: Sage Publications.

Ministerio de Sanidad, Servicios Sociales e lgualdad (2015). Plan estratégico de prevención y control de la infección por el VIH y otras infecciones de transmisión sexual 2013-2016. Ministerio de Sanidad, Servicios Sociales e Igualdad. Recuperado de http://www.msssi.gob.es/ ciudadanos/enfLesiones/enfTransmisibles/ sida/docs/PlanEstrategico2013 2016.pdf Morales, S., Fischman, R., Echávarri, O., Barros, J., Armijo, I., Moya, C., ... Núñez, C. (2016). Vivencia-expresión de la rabia y razones para vivir en un grupo de pacientes Chilenos con 
riesgo suicida. Revista Iberoamericana de Psicología y Salud, 7, 60-68. doi: 10.1016/i. rips. 2016.03 .00

Motofei, I.G., Rowland, D.L., Popa, F., Kreienkamp, D. y Paunica, S. (2011). Preliminary study with bicalutamide in heterosexual and homosexual patients with prostate cancer: A possible implication of androgens in male homosexual arousal. BJU International, 110-115. doi: 10.1111/i.1464-410X.2010.09764.x

Moyano, N., Monge, F. y Sierra, J.C. (2017). Predictors of sexual aggression in adolescents: Gender dominances vs. rape supportive attitudes. The European Journal of Psychology Applied to Legal Context, 9, 25-31. doi: 10.1016/i.ejpal.2016.06.001

Muñiz, J., Elosua, P. y Hambleton, R.K. (2013). Directrices para la traducción y adaptación de los tests: Segunda edición. Psicothema, 25, 151-157. doi: 10.7334/psicothema2013.24

Mustanski, B. y Liu, R.T. (2013). A longitudinal study of predictors of suicide attempts among lesbian, gay, bisexual, and transgender youth. Archives of Sexual Behavior, 42, 437-448. doi:10.1007/s10508-012-0013-9

National Heart, Lung y Blood Institute. (2014). Quality assessment tool for observational cohort and cross-sectional studies. National Hear, Lung, and Blood Institute. Recuperado de https://www.nhlbi.nih.gov/health-pro/ guidelines/in-develop/cardiovascular-riskreduction/tools/cohort

ONUSIDA. (2017). UNAIDS DATA 2017. ONUSIDA. Recuperado de htp://www. unaids.org/sites/default/files/media asset/20170720 Data book 2017 en.pdf

Organización Mundial de la Salud (2010). Measuring sexual health: Conceptual and practical considerations and related indicators. Recuperado de http://search.proquest.com/ docview/758117778? accountid=14542

Parsons, J.T., Starks, T.J., Gamarel, K.E. y Grov, C. (2012). Non-monogamy and sexual relationship quality among same-sex male couples. Journal of Family Psychology, 26, 669-677. doi: 10.1037/a0029561

Peplau, L.A. y Fingerhut, A. (2007). The close relationships of lesbians and gay men. Annual Review of Psychology, 58, 405-424. doi: 10.1 146/annurev.psych.58.110405.085701

Peplau, L.A., Fingerhut, A. y Beals, K.P. (2004). Sexuality in the relationships of lesbians and gay men. En J. Harvey, A. Wenzel y S. Sprecher (Eds.), Handbook of sexuality in close relationships (pp. 350-369). Mahwah, NJ: Erlbaum

Remien, R.H., Wagner, G., Dolezal, C. y CarballoDiéguez, A. (2003). Levels and correlates of psychological distress in male couples of mixed HIV status. AIDS Care 15, 525-538. doi: 10.1080/0954012031000134764

Reynolds III, C.F., Frank, E., Thase, M.E., Houck, P.R., Jennings, J.R., Jennings, J.R. ... Kupfer, D.J. (1988). Assessment of sexual function in depressed, impotent, and healthy men: Factor analysis of a brief sexual function questionnaire for men. Psychiatric Research, 24, 231-250. Doi: 10.1016/0165-1781 (88)90106-0

Rojas Castro, D., Le Gall, J.M., Andreo, C. y Spire, B. (2010). Stigma, discrimination, and sexual (dis) satisfaction among people living with HIV: Results from the "AIDES et toi" survey. AIDS Care, 22, 961-969. doi: 10.1080/09540121003758614

Rosen, R., Brown, C., Heiman, J., Leiblum, S., Meston, C., Shabsigh, R... D'Agostino, R. Jr. (2000). The Female Sexual Function Index (FSFI): A multidimensional self-report instrument for the assessment of female sexual function. Journal of Sex \& Marital Therapy, 26, 191-208. doi:10.1080/009262300278597

Rosen, R.C., Riley, A., Wagner, G., Osterloh, I.H., Kirkpatrick, J. y Mishra, A. (1997). The International Index of Erectile Function (IIFE): A multidimensional scale for assessment of erectyle dysfunction. Urology, 49, 822-830. doi: 10.1016/S0090-4295(97)00238-0

Rosenzweig, J.M. y Dailey, D.M. (1989). Dyadic adjustment/sexual satisfaction in women and men as a function of psychological sex-role selfperception. Journal of Sex \& Marital Therapy, 15, 42-56. doi: 10.1080/00926238908412846

Rosenzweig, J.M. y Lebow, W.C. (1992). Femme on the streets, butch in the sheets? Journal of Homosexuality, 23, 1-20. doi: 10.1300/ J082v23n03 01

Ross, M.W. y Rosser, B.R.S. (1996). Measurement and correlates of internalized homophobia: A factor analytic study. Journal of Clinical Psychology, 52, 15-21. doi: 10.1002/ (SICI) 1097-4679(199601)52:1<15::AIDJCLP2>3.0.CO;2-V

Rosser, B.R.S., Metz, M.E., Bockting, W.O. y Buroker, T. (1997). Sexual difficulties, concerns, and satisfaction in homosexual men: An empirical study with implications for HIV prevention. 
Journal of Sex \& Marital Therapy, 23, 61 -73. doi: 10.1080/00926239708404418

Rowen, C.J. y Malcolm, J.P. (2003). Correlates of internalized homophobia and homosexual identity formation in a sample of gay men. Journal of Homosexuality, 43, 77-92. doi: 10.1300/J082v43n02 05

Ruiseñor-Escudero, H., Grosso, A., Ketende, S., Pitche, V., Simplice, A., Tchalla, J., ... Baral, S. (2017). Using a social ecological framework to characterize the correlates of HIV among men who have sex with men in Lomé, Togo. AIDS Care, 29, 1169-1177. doi: 10.1080/09540121.2017.1280122

Rzeszutek, M., Oniszczenko, W., Schier, K., BiernatKałuža, E. y Gasik, R. (2016). Temperament traits, social support, and trauma symptoms among HIV/AIDS and chronic pain patients. International Journal of Clinical and Health Psychology, 16, 137-146. doi: 10.1016/i. iichp.2015.10.001

Sánchez, D.T., Moss-Racusin, C.A., Phelan, J.E. y Crocker, J. (2011). Relationship contingency and sexual motivation in women: Implications for sexual satisfaction. Archives of Sexual Behavior, 40, 99-110. doi: 10.1007/s10508009-9593-4

Sánchez-Fuentes, M.M., Salinas, J.M. y Sierra, J.C. (2016). Use of an ecological model to study sexual satisfaction in a heterosexual Spanish sample. Archives of Sexual Behavior, 45, 1973-1988. doi: 10.1007/s10508-0160703-9

Sánchez-Fuentes, M.M., Santos-Iglesias, P. y Sierra, J.C. (2014). A systematic review of sexual satisfaction. International Journal of Clinical and Health Psychology, 14, 67-75. doi: 10.1016/S1697-2600(14)70038-9

Sánchez-Fuentes, M.M. y Sierra, J.C. (2015). Sexual satisfaction in a heterosexual and homosexual Spanish sample: The role of socio-demographic characteristics, health indicators, and relational factors. Sexual and Relationship Therapy, 30, 226-242. doi: 10.1080/14681994.2014.978275

Sandfort, T.G.M., de Graaf, R. y Biil, R.V. (2003). Same-sex sexuality and quality of life: Findings from the Netherlands Mental Health Survey and Incidence Study. Archives of Sexual Behavior, 32, 15-22. doi: 10.1023/A: 1021885127560 Santos-Iglesias, P. y Sánchez-Fuentes, M.; (2016). Sexual satisfaction in Spanish heterorsexual couples: Testing the Interpersonal Exchange Model of Sexual Satisfaction. Journal of Sex \& Marital Therapy, 42, 223-242. doi: 10.1080/0092623X.2015.1010675

Schönfeld, P., Brailovskaia, J., Bieda, A., Chi Zhang, X. y Margraf, J. (2016). The effects of daily stress on positive and negative mental health: Mediation through self-efficacy. International Journal of Clinical and Health Psychology, 16, 1-10. doi: 10.1016/i.ijchp.2015.08.005

Schwartz, P. y Young, L. (2009). Sexual Satisfaction in Committed Relationships. Sexuality Research and Social Policy, 6, 1-17. doi: 10.1525/ srsp.2009.6.1.1

Šević, S., Ivanković, I. y Štulhofer, A. (2016). Emotional intimacy among coupled heterosexual and gay/bisexual Croatian men: Assessing the role of minority stress. Archives of Sexual Behavior, 45, 1259-1268. doi: $10.1007 / \mathrm{s} 10508-015-0538-9$

Sierra, J.C. y Buela-Casal, G. (2004). Evaluación y tratamiento de las disfunciones sexuales. En G. Buela-Casal y J. C. Sierra (Eds.), Manual de evaluación y tratamiento psicológicos $\left(2^{a}\right.$ ed., pp. 439-485): Madrid: Biblioteca Nueva.

Sierra, J.C., Moyano, N., Vallejo-Medina, P. y Gómez-Berrocal, C. (2018). An abridged Spanish version of Sexual Double Standard Scale: Factorial stucture, reliability and validity evidence. International Journal of Clinical and Health Psychology, 18, 69-80. doi: 10.1016/i. iichp.2017.05.003

Smith, B.L. y Horne, S.G. (2008). What's faith got to do with it? The role of spirituality and religion in lesbian and bisexual women's sexual satisfaction. Women \& Therapy, 31, 73-87. doi: 10.1300/02703140802145243

Snell, W. E. (1998). The multidimensional sexual self-concept questionnaire. En C. M. Davis, W. L. Yarber, R. Bauserman, G. Schreer y S. L. Davis (Eds.), Handbook of sexuality-related measures (pp. 521-524). Thousand Oaks, CA: Sage.

Snell, W.E., Fisher, T.D. y Walters, A.S. (1993). The Multidmensional Sexuality Questionnaire: An objective self-report measure of psychological tendencies associated with human sexuality. Annals of Sex Research, 6, 27-55. doi: 10.1007/BF00849744

Spanier, G.B. (1976). Measuring dyadic adjustment. Journal of Marriage and the Family, 38, 15-28. doi: 10.2307/350547 
Sprecher, S. (2002). Sexual satisfaction in premarital relationships: Associations with satisfaction, love, commitment, and stability. Journal of Sex Research, 39, 190-196. doi: 10.1080/00224490209552141

Starks, T.J., Gamarel, K.E. y Johnson, M.O. (2014). Relationship characteristics and HIV transmission risk in same-sex male couples in HIV serodiscordant relationships. Archives of Sexual Behavior, 43, 139-147. doi: 10.1007/ s10508-013-0216-8

Starks, T.J., Grov, C. y Parsons, J.T. (2013). Sexual compulsivity and interpersonal functioning: Sexual relationship quality and sexual health in gay relationships. Health Psychology, 32, 1047-1056. doi: 10.1037/a0030648

Stern, S. y Wright, J. (2017). Discrete effects of religiosity and spirituality on gay identity and self-esteem. Journal of Homosexuality, 65, 1071-1092. doi: 10.1080/00918369.2017.1368769

Štulhofer, A., Buško, V. y Brouillard, P. (201 1). The New Sexual Satisfaction Scale and its short form. En T.D. Fisher, C.M. Davis, W.L. Yarber y S.L. Davis (Eds.), Handbook of sexuality related measures ( $3^{a}$ ed., pp. 530-532). Nueva York, NY: Routledge.

Thoits, P. A. (1995). Stress, coping, and social support processes: Where are we? What next? Journal of Health and Social Behavior, Special number, 53-79. doi: 10.2307/2626957
Totenhagen, C.J., Butler, E.A. y Ridley, C.A. (2012). Daily stress, closeness, and satisfaction in gay and lesbian couples. Personal Relationships, 19, 219-233. doi: 10.1111/i.1475$\underline{6811.2011 .01349 . x}$

Tracy, J.K. y Junginger, J. (2007). Correlates of lesbian sexual functioning. Journal of Women's Health, 16, 499-509. doi: 10.1089/ iwh.2006.0308

Weingourt, R. (1998). A comparison of heterosexual and homosexual long-term sexual relationships. Archives of Psychiatric Nursing, 12, 114-118. doi: 10.1016/S0883$9417(98) 80061-7$

Whitley, M.P. (1998). Sexual Satisfaction Inventory. En C. M. Davis, W. L. Yarber, R. Bauserman, G. Schreer y S. L. Davis (Eds.), Handbook of sexuality-related measures (pp. 519-521). Thousand Oaks, CA: Sage.

Zea, M.C., Olaya, P., Reisen, C.A. y Poppen, P.J. (2016). MSM in Bogotá are living with HIV for extended periods without diagnosis or treatment. International Journal of STD and AIDS, 28, 920-924. doi: $10.1177 / 0956462416681364$

Zheng, L. y Zheng, Y. (2017). Sexual satisfaction in Chinese gay and bisexual men: Relationship to negative sexual minority identity and sexual role preference. Sexual and Relationship Therapy, 32, 75-88. http://doi.org/10.1080/ 14681994.2016 .1200027$. 